\title{
U so do sistema de resfriamento adiabático evaporativo no conforto térmico de vacas da raça girolando ${ }^{1}$
}

\author{
G ledson L. P. de Almeida ${ }^{2}$, Héliton Pandorfi ${ }^{3}$, Cristiane G uiselini ${ }^{3}$, \\ H ugo M. Henrique ${ }^{3}$ \& G leidiana A. P. de Almeida ${ }^{4}$
}

\begin{abstract}
RESU MO
O bjetivou-se, com esta pesquisa, avaliar diferentes tempos de exposição dos animais à climatização no curral de espera sobre os índices de conforto, parâmetros fisiológicos e produção de leite de vacas girolando 7/8. Consideraram-se tratamentos quatro tempos de exposição dos animais ao sistema de resfriamento adiabático evaporativo (SRAE), 0, 10, 20 e $30 \mathrm{~min}$. 0 experimento foi realizado durante a estação de verão, com duração de 56 dias. U tilizaram-se 16 vacas com produção média de $18 \mathrm{~kg}$ de leite $\mathrm{d}^{-1}$, adotando-se delineamento em quadrado latino $4 \times 4$. A temperatura de bulbo seco $\left(\mathrm{Tbs},{ }^{\circ} \mathrm{C}\right)$ e a umidade relativa ( $U R, \%)$, foram registradas a cada minuto o que permitiu determinar a eficiência do SRAE por meio do índice de temperatura e umidade (ITU) e entalpia (h). A frequência respiratória (FR), temperatura retal (TR) e temperatura de pelame (TP), foram medidas antes e depois da climatização. 0 tratamento 30 min permitiu manter as variáveis ambientais e os índices de conforto dentro dos limites recomendados. As variáveis fisiológicas (FR, TR e TP) mostraram valores inferiores no tratamento $30 \mathrm{~min}$, o que refletiu positivamente na produção de leite, com aumento de $4,4 \%$, quando comparado com 0 tratamento $0 \mathrm{~min}$
\end{abstract}

Palavras-chave: ambiência, biometeorologia animal, bovinos leiteiros

\section{U se of adiabatic evaporative cooling system in thermal comfort of Girolando cows}

\begin{abstract}
The objective of this research was to evaluate different times of exposure of animals to cooling in the waiting pen on the comfort index, physiological parameters and milk production of girolando $7 / 8$ cows. As treatments, four times of exposure of animals to adiabatic evaporative cooling system (AECS), $0,10,20$ and $30 \mathrm{~min}$ were considered. The experiment was conducted during the summer season and lasted 56 days. Sixteen cows were used with an average daily milk production of $18 \mathrm{~kg}$, distributed in $4 \times 4$ Latin square design. The dry bulb temperature (DBT) and relative humidity (RH) were recorded every minute, which allowed to determine the efficiency of the AECS through the Temperature and Humidity Index (THI) and enthalpy (h). The respiratory rate (RR), rectal temperature (RT) and temperature of the coat (TC) were measured before and after cooling. The $30 \mathrm{~min}$ treatment kept the environmental variables and the comfort indexes within recommended limits. The physiological variables (RR, RT and TC) were lower in the $30 \mathrm{~min}$ treatment and reflected positively on milk production, which increased $4.4 \%$ compared to the 0 min treatment.
\end{abstract}

Key words: ambience, animal biometeorology, dairy cattle

\footnotetext{
${ }^{1}$ Parte da Dissertação de Mestrado do primeiro autor

2 Mestre em Engenharia Agrícola/U FRPE, Av. D om Manoel de Medeiro, D ois Irmãos, CEP 52171-900, Recife, PE. Fone: (81) 3320-6279. E-mail: gledson@dtr.ufrpe.br

${ }^{3}$ DTR/U FRPE. Fone: (81) 3320-6260. E-mail: pandorfi@dtr.ufrpe.br; guiselini@dtr.ufrpe.br; hugoufrpe@hotmail.com

${ }^{4}$ U AG/U FRPE. Av. Bom Pastor s/n, CEP 55296-901, Garanhuns, PE. Fone: (87) 3761-0882. E-mail: amelinhasbu@hotmail.com
} 


\section{INTRODUÇÃO}

Os fatores ambientais externos podem interferir no microclima gerado no interior das instalações, causando impacto sobre a produção, com consequentes prejuízos econômicos para os produtores e indústrias do setor leiteiro de vários países.

Uma forma de avaliar as respostas dos animais ao ambiente térmico é através da observação de alguns parâmetros fisiológicos, como a temperatura retal e a frequência respiratória. Esses parâmetros podem ser influenciados por fatores intrínsecos (idade, raça, estado fisiológico), e por fatores extrínsecos (hora do dia, ingestão de alimentos e de água, temperatura ambiente, velocidade do vento, estação do ano), segundo Perissinotto et al. (2009).

Em ambientes tropicais a magnitude do estresse térmico é causada pelo efeito combinado de alta temperatura e umidade relativa do ar, elevada incidência de radiação solar e baixa velocidade do vento, o que reduz a eficiência da perda de calor (Dikmen \& Hansen, 2009) limitando o desenvolvimento, a produção e a reprodução dos animais (Avendano et al., 2006).

O ambiente é considerado confortável quando o animal se encontra em equilíbrio térmico, ou seja, o calor produzido pelo metabolismo é perdido para o ambiente sem prejuízo da homeostase do animal (Silva et al., 2010).

A temperatura ótima para exploração leiteira depende da espécie, raça, idade, consumo alimentar, aclimatação, nível de produção, pelame e grau de tolerância do animal ao calor e ao frio. A raça holandesa, especializada em produção leiteira, possui zona de termoneutralidade situada entre 4 e $26^{\circ} \mathrm{C}$ (Huber, 1990), em que a homeotermia é mantida indiretamente pelos processos de transferência de calor por radiação, convecção, condução e evaporação que ocorrem na superfície do animal (Azevedo et al., 2005).

Para minimizar os efeitos do estresse térmico, em particular sobre a produção de leite, pode-se adotar tecnologias que possibilitem o manejo estratégico do rebanho atendendo às exigências nutricionais e com a utilização de raças adaptadas ao ambiente (Souza et al., 2003). A movimentação e o resfriamento adiabático evaporativo do ar e o uso de sombra para minimizar o efeito da radiação solar, são artifícios empregados para potencializar a dissipação de calor (West, 2003).

Objetivou-se, com esta pesquisa, avaliar diferentes tempos de exposição dos animais à climatização no curral de espera sobre os índices de conforto, parâmetros fisiológicos e produção de leite de vacas da raça girolando.

\section{MATERIAL E MÉTODOS}

A pesquisa foi realizada em uma propriedade comercial de produção de leite tipo B, Fazenda Roçadinho, no município de Capoeiras, PE, latitude de $8^{\circ} 36^{\prime} 33^{\prime \prime} \mathrm{S}$, longitude de $36^{\circ} 37^{\prime} 30^{\prime \prime}$ O e altitude de $733 \mathrm{~m}$. O índice pluviométrico da região é de 588 $\mathrm{mm}$ por ano e temperatura média anual de $22,1^{\circ} \mathrm{C}$. De acordo com a classificação climática de Köppen, o clima da região é caracterizado como Bsh, semiárido.
O experimento ocorreu durante a estação de verão (fevereiro a março de 2009), com duração de 56 dias divididos em quatro períodos (P1, P2, P3 e P4), totalizando 14 dias para cada período experimental, utilizando-se os sete primeiros dias de cada período para adaptação dos animais aos tratamentos; após a adaptação foram registradas as variáveis meteorológicas em cada tratamento e as variáveis externas à instalação, dados fisiológicos e de produção.

Consideraram-se tratamentos quatro tempos de climatização no curral de espera, $0,10,20$ e $30 \mathrm{~min}$, no qual os animais foram expostos ao sistema de resfriamento adiabático evaporativo (SRAE) na pré-ordenha.

A seleção dos animais do experimento contou com 16 vacas girolando multíparas, em lactação, com composição genética de 7/8 Holandês-Gir, com peso médio de $500 \mathrm{~kg}$ e produção média de leite de $18 \mathrm{~kg} \mathrm{~d}^{-1}$ sendo, então, divididas ao acaso, em quatro grupos (G1, G2, G3 e G4) com quatro animais para cada grupo.

O curral de espera apresentava dimensões de 3,0 $\mathrm{m}$ de pédireito, $8 \mathrm{~m}$ de largura e $6 \mathrm{~m}$ de comprimento $\left(48 \mathrm{~m}^{2}\right)$, com piso de pedra rejuntada com pasta de cimento. A cobertura foi feita com malha preta de sombreamento $(70 \%)$, disposta em camada única sobre estrutura de madeira, sem fechamento lateral.

A composição do SRAE contou com dois ventiladores axiais da marca Ventiave, modelo P3D-Plus, equipados com motor trifásico de $0,5 \mathrm{HP}$, com diâmetro de $1,0 \mathrm{~m}$, vazão de $240 \mathrm{~m}^{3} \mathrm{~min}^{-1}$, 965 RPM, e capacidade de produzir movimentação de ar de até $2,5 \mathrm{~m} \mathrm{~s}^{-1}$, instalados com espaçamento de $6 \mathrm{~m}$ entre equipamentos; a altura de $2,5 \mathrm{~m}$ do piso e inclinação, em relação à vertical, foi de $20^{\circ}$.

O sistema de nebulização foi composto de cinco linhas (tubo de polietileno) com quatro bicos nebulizadores por linha, com espaçamento de 1,5 m entre bicos e entre linhas a 3,0 $\mathrm{m}$ de altura do piso. Este sistema foi equipado com uma bomba centrífuga da marca Schneider ${ }^{\circledR}$, modelo BC-92SK, motor trifásico de $0,75 \mathrm{CV}$, consumo de energia de $0,65 \mathrm{KW} \mathrm{h}^{-1} \mathrm{e}$ vazão de $240 \mathrm{~L} \mathrm{~h}^{-1}$. O SRAE era acionado manualmente e permanecia ligado ininterruptamente, durante a permanência dos animais no curral de espera.

As variáveis meteorológicas foram registradas a cada minuto, no curral de espera e no ambiente externo, durante a permanência dos animais neste curral, por meio de dataloggers modelo HOBO Pro HB8 (Onset Computer Corporation Bourne, MA, USA) para o registro da temperatura de bulbo seco (Tbs) e da umidade relativa do ar (UR\%).

Para determinação da eficiência térmica do SRAE no curral de espera, determinaram-se o índice de temperatura e a umidade (ITU) proposto por Thom (1959) e entalpia ( $\mathrm{h} ; \mathrm{KJ} \mathrm{kg}^{-1}$ ) proposta por Albright (1990) pelas Eqs. 1, 2 e 3:

$$
\mathrm{ITU}=\mathrm{Tbs}+0,36 \text { Tpo }+41,5
$$

em que:

Tbs - temperatura do bulbo seco, ${ }^{\circ} \mathrm{C}$

Tpo - temperatura de ponto de orvalho, ${ }^{\circ} \mathrm{C}$

$$
\mathrm{h}=1,006 \mathrm{Tbs}+\mathrm{W}(2501+1,805 \mathrm{Tbs})
$$


em que:

Tbs- temperatura de bulbo seco, ${ }^{\circ} \mathrm{C}$

$\mathrm{W}$ - razão de mistura, $\mathrm{kg}$ vapor d'água $\mathrm{kg}$ ar seco ${ }^{-1}$

$$
\mathrm{W}=\frac{0,622 \mathrm{ea}}{\mathrm{P}-\mathrm{ea}}
$$

em que:

ea - pressão atual de vapor d'água, $\mathrm{KPa}$

$\mathrm{P}$ - pressão atmosférica, $\mathrm{KPa}$

Os parâmetros fisiológicos, temperatura retal $\left(\mathrm{TR},{ }^{\circ} \mathrm{C}\right)$,

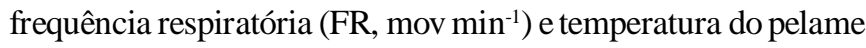
$\left(\mathrm{TP},{ }^{\circ} \mathrm{C}\right)$ foram registrados nos dois turnos de ordenha, das 5 às $5 \mathrm{~h} 30 \mathrm{~min}$ e das 14 às $14 \mathrm{~h} 30 \mathrm{~min}$, duas vezes por semana, antes e depois da climatização.

Determinou-se a temperatura média do pelame (TP), de acordo com Pinheiro et al. (2005), com registros de temperatura da cabeça, dorso, canela e úbere de cada animal, em seus respectivos tratamentos, por meio da Eq. 4:

$$
\mathrm{TP}=0,01 \mathrm{~T}_{\text {cabeça }}+0,07 \mathrm{~T}_{\text {dorso }}+0,12 \mathrm{~T}_{\text {canela }}+0,08 \mathrm{~T}_{\text {úbere }}
$$

A produção de leite (PL) foi quantificada individualmente para cada animal em seu respectivo tratamento, para as duas ordenhas diárias (manhã e tarde).

O delineamento experimental adotado foi o quadrado latino $4 \times 4$, conforme Tabela 1. Para a análise das variáveis estudadas (meteorológicas, índices de conforto, fisiológicas e produção) utilizou-se o software Statistcal Analysis System (SAS, 1997) e as inferências obtidas foram avaliadas pelo teste de Tukey $(\mathrm{P}<0,05)$.

Tabela 1. Representação esquemática do delineamento

\begin{tabular}{|c|c|c|c|c|c|c|c|c|}
\hline & \multicolumn{2}{|c|}{ P1 } & \multicolumn{2}{|c|}{ P2 } & \multicolumn{2}{|c|}{ P3 } & \multicolumn{2}{|c|}{ P4 } \\
\hline & $7 \mathrm{~d}$ & $7 \mathrm{~d}$ & $7 \mathrm{~d}$ & $7 \mathrm{~d}$ & $7 \mathrm{~d}$ & $7 \mathrm{~d}$ & $7 \mathrm{~d}$ & $7 \mathrm{~d}$ \\
\hline $\begin{array}{l}\text { G1 } \\
\text { G2 } \\
\text { G3 } \\
\text { G4 }\end{array}$ & $\begin{array}{l}\frac{8}{80} \\
\frac{8}{8} \\
\frac{8}{8} \\
\frac{8}{8}\end{array}$ & $\begin{array}{l}\text { T1 } \\
\text { T2 } \\
\text { T3 } \\
\text { T0 }\end{array}$ & $\begin{array}{l}\frac{80}{0} \\
\frac{9}{80} \\
\frac{8}{8}\end{array}$ & $\begin{array}{l}\text { T0 } \\
\text { T1 } \\
\text { T2 } \\
\text { T3 }\end{array}$ & $\frac{\sqrt[8]{4}}{\frac{\sqrt{9}}{\frac{0}{8}}}$ & $\begin{array}{l}\text { T3 } \\
\text { T0 } \\
\text { T1 } \\
\text { T2 }\end{array}$ & $\frac{\sqrt[8]{4}}{\frac{\sqrt{9}}{\frac{0}{8}}}$ & $\begin{array}{l}\text { T2 } \\
\text { T3 } \\
\text { T0 } \\
\text { T1 }\end{array}$ \\
\hline
\end{tabular}
estatístico utilizado no experimento

P - período; G - grupo; T - tratamentos; d - dias

\section{RESULTADOS E DISCUSSÃO}

Verifica-se, na Tabela 2, que o valor médio para Tbs registrado no tratamento $30 \mathrm{~min}$ (turno da manhã), apresentou redução significativa $(\mathrm{P}<0,05)$, quando comparado com os tratamentos 0 e $10 \mathrm{~min}$; entretanto, todos se mantiveram dentro da zona de conforto térmico (ZCT) com temperatura do ar entre 4 e $26{ }^{\circ} \mathrm{C}$, considerada por Huber (1990), adequada para o conforto térmico de vacas em lactação. O tratamento 30 min mostrou-se eficiente na redução desta variável, com valores da ordem de 1,$5 ; 0,7$ e $0,2{ }^{\circ} \mathrm{C}$, quando comparado com os tratamentos 0,10 e $20 \mathrm{~min}$, respectivamente. Nota-se, no turno da tarde, que o sistema de climatização foi eficiente na redução da temperatura ambiente, apresentando diferenças significativas $(\mathrm{P}<0,05)$ entre os tratamentos 20 e $30 \mathrm{~min}$, que ficaram abaixo de $26^{\circ} \mathrm{C}$; os tratamentos 0 e $10 \mathrm{~min}$ superaram o limite crítico superior em 3,2 e $0,4^{\circ} \mathrm{C}$.

A Tbs observada no curral de espera climatizado corrobora com estudo realizado por Arcaro Júnior et al. (2005), que verificaram redução na Tbs, às $7 \mathrm{~h}$ de $0,2^{\circ} \mathrm{C}$ e às $14 \mathrm{~h}$ de $3,5^{\circ} \mathrm{C}$ no interior de freestall equipado com SRAE, para vacas lactantes, quando comparado com freestall sem climatização.

Os valores de UR registrados no turno da manhã (Tabela 2) apresentaram diferenças $(\mathrm{P}<0,05)$; entretanto, todos os tratamentos se encontravam acima de $70 \%$, considerado valor limite para o conforto de vacas lactantes em clima quente (Nääs \& Arcaro Júnior, 2001). No turno da tarde a UR registrada nos tratamentos com climatização aumentou significativamente $(\mathrm{P}$ $<0,05)$ com o tempo de funcionamento do SRAE, estando os tratamentos 20 e 30 min superiores a $70 \%$, acima, portanto, da zona de conforto térmico, entre 50 e $70 \%$, segundo recomendações de Tinôco (2001); apesar disto, para Perissinotto \& Moura (2007), quando a Tbs se encontra próximo ao limite superior da zona de termoneutralidade $\left(26^{\circ} \mathrm{C}\right)$, independente dos valores de UR, a sensação de conforto térmico para vacas em lactação é muito boa.

Arcaro Júnior et al. (2005) verificaram umidade relativa elevada com emprego do SRAE aplicado no curral de espera. Os valores encontrados foram 45,6; 38,9 e 79,8\%, respectivamente, para os tratamentos controle, ventilação e ventilação associada à nebulização.

Constatou-se redução significativa $(\mathrm{P}<0,05)$ no ITU para o tratamento $30 \mathrm{~min}$, quando comparado com os tratamentos 0 ,

Tabela 2. Valores médios e desvio padrão das variáveis ambientais e dos índices de conforto térmico registrados no

\begin{tabular}{|c|c|c|c|c|c|}
\hline \multirow{2}{*}{ Variáveis } & \multicolumn{4}{|c|}{ Tratamentos (min) } & \multirow{2}{*}{ CV $(\%)$} \\
\hline & 0 & 10 & 20 & 30 & \\
\hline \multicolumn{6}{|c|}{ Turno da manhã } \\
\hline $\operatorname{Tbs}\left({ }^{\circ} \mathrm{C}\right)$ & $21,4 a \pm 1,51$ & $20,6 b \pm 1,04$ & $20,1 b c \pm 0,84$ & $19,9 c \pm 0,78$ & 05,58 \\
\hline UR $(\%)$ & $86,5 c \pm 5,78$ & $90,3 b \pm 3,63$ & $92,5 a b \pm 2,43$ & $93,5 a \pm 1,96$ & 05,06 \\
\hline ITU & $70,0 a \pm 1,55$ & $69,0 b \pm 1,22$ & $68,8 b \pm 1,04$ & $68,0 c \pm 0,99$ & 01,93 \\
\hline $\mathrm{h}\left(\mathrm{KJ} \mathrm{kg} \mathrm{kg}^{-1}\right)$ & $60,4 a \pm 2,98$ & $59,3 b \pm 2,49$ & $58,7 b c \pm 2,33$ & $58,4 c \pm 2,28$ & 04,40 \\
\hline \multicolumn{6}{|c|}{ Turno da tarde } \\
\hline $\operatorname{Tbs}\left({ }^{\circ} \mathrm{C}\right)$ & $29,2 a \pm 2,99$ & $26,4 b \pm 2,10$ & $25,0 c \pm 1,52$ & $24,3 d \pm 1,25$ & 10,63 \\
\hline UR $(\%)$ & $57,3 d \pm 15,94$ & $69,5 c \pm 12,73$ & $74,8 b \pm 10,62$ & $77,5 a \pm 9,60$ & 20,79 \\
\hline ITU & $77,8 a \pm 2,66$ & $75,3 b \pm 1,97$ & $73,5 c \pm 1,44$ & $73,0 \mathrm{c} \pm 1,26$ & 03,51 \\
\hline $\left.\mathrm{h}(\mathrm{KJ} \mathrm{kg})^{-1}\right)$ & $70,5 a \pm 4,13$ & $68,7 b \pm 3,62$ & $66,9 c \pm 3,55$ & $65,8 d \pm 3,65$ & 05,87 \\
\hline
\end{tabular}
interior do curral de espera, nos turnos manhã e tarde

Médias seguidas das mesmas letras nas mesmas linhas não diferem entre si a nível de $5 \%$ de probabilidade, pelo teste de Tukey 
10 e 20 min (Tabela 2), verificando-se que no turno da manhã o ambiente apresentou condições ideais para vacas mestiças em lactação de composição genética 7/8 Holandês-Gir que, segundo Azevedo et al. (2005), o ITU deve ser $<75$. O acondicionamento no interior do curral de espera foi próximo ao obtido por Silva et al. (2002), que encontraram ITU de 72,9 no turno da manhã em curral de espera equipado com SRAE para vacas em lactação.

No turno da tarde a climatização do ambiente proporcionou redução significativa $(\mathrm{P}<0,05)$ para o ITU nos tratamentos 20 e $30 \mathrm{~min}$, comparativamente com 0 e 10 min (Tabela 2), que estiveram acima do valor considerado limite crítico superior (ITU < 75) para vacas 7/8 Holandês-Gir (Azevedo et al., 2005), em 2,8 e 0,3 unidades, respectivamente. Os resultados apontados no turno da tarde estão próximos aos observados por Silva et al. (2002) e Arcaro Júnior et al. (2005), que obtiveram ITU de 75,5 e 74,9, trabalhando com vacas em lactação no curral de espera, com tempos de exposição de 40 e $30 \mathrm{~min}$, respectivamente.

Os valores médios da quantidade de energia presente na parcela de ar, expressa pela entalpia, apontam redução significativa $(\mathrm{P}<0,05)$ no turno da manhã para os tratamentos 10, 20 e $30 \mathrm{~min}$, quando comparados com 0 min (Tabela 2). Todos os tratamentos apresentam entalpia abaixo do limite crítico superior de $67,4 \mathrm{KJ} \mathrm{kg}^{-1}$ de ar seco, obtido para temperatura de bulbo seco de $26^{\circ} \mathrm{C}$ e umidade relativa do ar de $70 \%$; mesmo assim, o tratamento 30 min mostrou-se eficiente na redução desta variável, com valores da ordem de 2,0; 0,9 e $0,3 \mathrm{KJ} \mathrm{kg}^{-1}$ de ar seco, frente aos valores encontrados nos tratamentos 0,10 e $20 \mathrm{~min}$, respectivamente.

O sistema de climatização, no turno da tarde, promoveu redução significativa $(\mathrm{P}<0,05)$ na entalpia entre os tratamentos $0,10,20$ e $30 \mathrm{~min}$. Os tratamentos $20 \mathrm{~min}\left(66,9 \mathrm{KJ} \mathrm{kg}^{-1} \mathrm{de}\right.$ ar seco) e $30 \mathrm{~min}\left(65,8 \mathrm{KJ} \mathrm{kg}^{-1}\right.$ de ar seco) permaneceram abaixo do valor crítico superior $\left(67,4 \mathrm{KJ} \mathrm{kg}^{-1}\right.$ de ar seco). As médias de entalpia observadas neste estudo para o turno da tarde estão abaixo das obtidas por Martello et al. (2004), que verificaram, às $13 \mathrm{~h}$, valores de 74,1; 71,2 e 70,8 $\mathrm{KJ} \mathrm{kg}^{-1}$ de ar seco, para instalações sem climatização, com climatização por ventilação e aspersão e instalação com tela de sombreamento, respectivamente.

Segundo Carvalho et al. (2009), regiões com altos valores de temperatura do ar associados aos das altas umidades relativas, são limitantes ao uso de sistemas de resfriamento evaporativo, porém durante os meses mais quentes e menos úmidos os sistemas de resfriamento evaporativo, apresentam melhor desempenho.

Observa-se, na Figura 1 (A), que a variação da Tbs em todos os tratamentos, incluindo o ambiente externo, atendeu à exigência térmica dos animais, permanecendo entre o limite crítico inferior de $0{ }^{\circ} \mathrm{C}$, segundo Pereira (2005) e superior a 26 ${ }^{\circ} \mathrm{C}$, de acordo com Huber (1990), para zona de conforto térmico (ZCT), demonstrando que a condição de conforto no curral de espera foi atendida, independente da climatização e do tempo de exposição dos animais ao SRAE. Verifica-se, porém, na Figura 1 (B), que os tratamentos 0, 10 e 20 min de exposição ao SRAE apresentaram valores da ordem de 86, 61 e $25 \%$ dos dias de estudo fora da faixa de conforto recomendada, enquanto no tratamento $30 \mathrm{~min}$ as temperaturas registradas no interior do curral de espera estiveram abaixo do limite crítico superior (26 $\left.{ }^{\circ} \mathrm{C}\right)$.

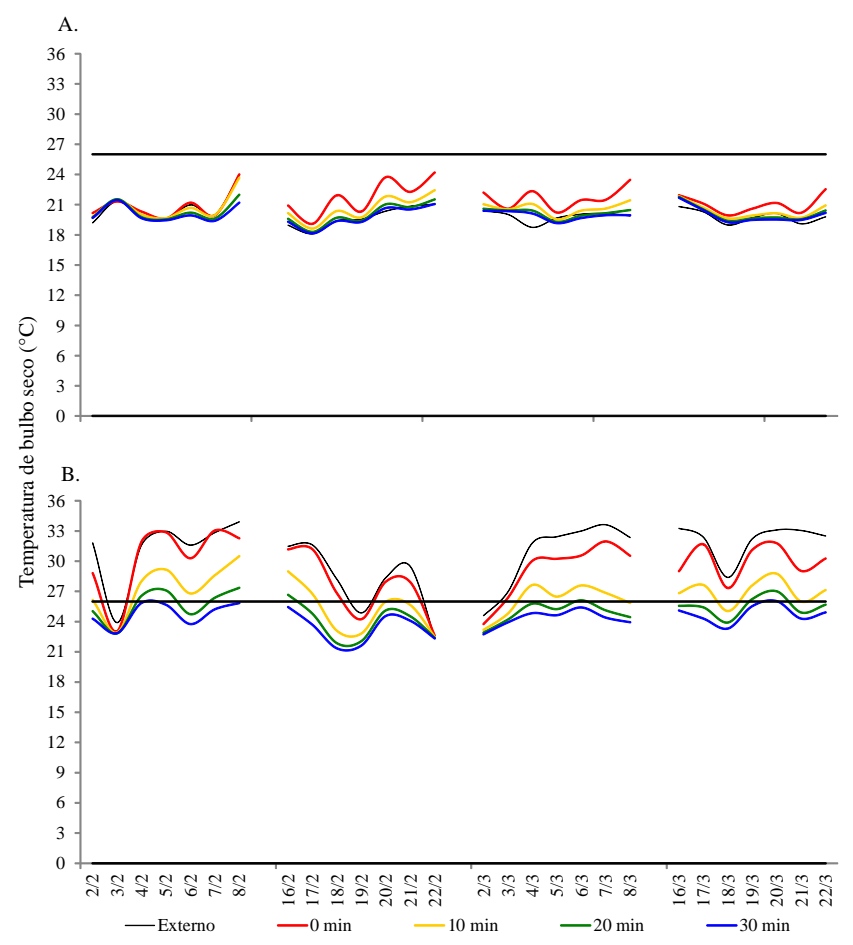

Figura 1. Variação da temperatura de bulbo seco nos diferentes tratamentos e no abrigo meteorológico (ambiente externo) no turno da manhã (A) e da tarde (B)

Observa-se, na Figura 2, a variação do índice de temperatura e umidade nos turnos da manhã (A) e da tarde (B). Esses indicativos demonstram que a condição de conforto no curral de espera foi atendida em todos os tratamentos no turno da manhã; no entanto se nota, para o turno da tarde, no tratamento $10 \mathrm{~min}$, que em $50 \%$ dos dias estudados o valor de ITU esteve acima de 75, enquanto no tratamento $20 \mathrm{~min}$, apenas $7 \%$. O único tratamento que condicionou o curral de espera dentro dos limites estabelecidos (64 a 75), durante toda a etapa experimental, foi aquele em que os animais permaneceram 30 min submetidos à climatização.

Os resultados da variação de entalpia apontam que a condição de conforto no curral de espera foi atendida em todos os tratamentos, no turno da manhã (Figura 3A); no entanto, para o turno da tarde (Figura 3B), nos tratamentos 0 e $10 \mathrm{~min}$, em $61 \%$ dos dias estudados o valor da entalpia esteve acima do limite crítico $\left(67,4 \mathrm{KJ} \mathrm{kg}^{-1}\right.$ de ar seco); no tratamento $20 \mathrm{~min}$ em $53 \%$, enquanto no tratamento em que os animais ficaram submetidos durante 30 min à climatização, apresentou $32 \%$ dos dias avaliados acima do valor recomendado.

As variações da entalpia observadas neste estudo para o tratamento 30 min no turno da tarde, corroboram com Araujo (2001) que verificou, no turno da tarde (13 h), 27,8\% dos dias avaliados valores acima do limite crítico $\left(67,4 \mathrm{KJ} \mathrm{kg}^{-1}\right.$ de ar seco) para instalações do tipo freestall com sistema de climatização, para vacas da raça holandesa em lactação. Ainda segundo Araújo (2001), esse resultado demonstra a capacidade 


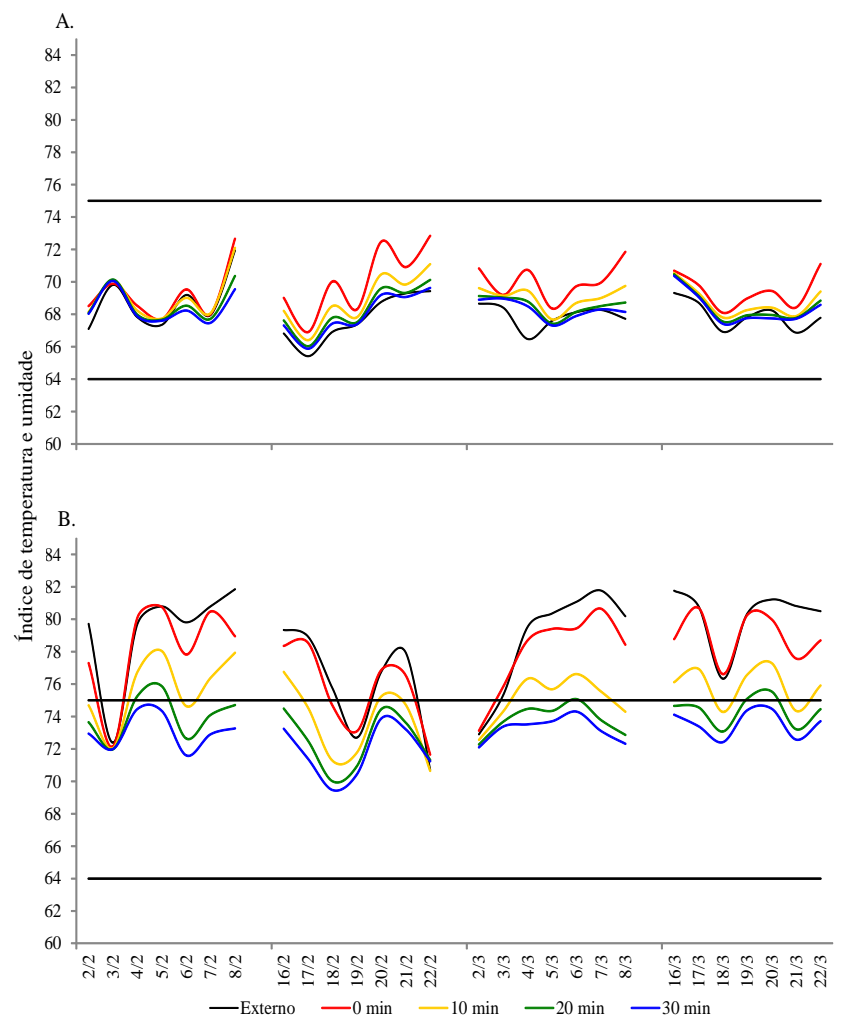

Figura 2. Variação do índice de temperatura e umidade nos diferentes tratamentos e no abrigo meteorológico (ambiente externo) no turno da manhã (A) e tarde (B)

dessas instalações funcionarem como proteção contra a radiação solar e, provavelmente, o fato de terem ocorrido valores desconfortantes para os animais se deve ao manejo incorreto dos instrumentos de apoio, como ventilador e nebulizador, pois não existia um controle preciso da temperatura e umidade ambiente para serem ligados ou desligados.

A análise das médias para FR no turno da manhã (Tabela 3) não apontou diferença significativa $(\mathrm{P}>0,05)$ entre os animais submetidos aos tratamentos 10,20 e 30 mas se verifica efeito significativo $(\mathrm{P}<0,05)$ quando comparados com o tratamento 0 min, apresentando valores da ordem de 27,8; 28,0; 25,8 e 36,0 mov min $^{-1}$, respectivamente, embora tais valores de FR para vacas em lactação sejam considerados normais, entre 18 e 60 mov $\min ^{-1}$, de acordo com Hahn et al. (1997).

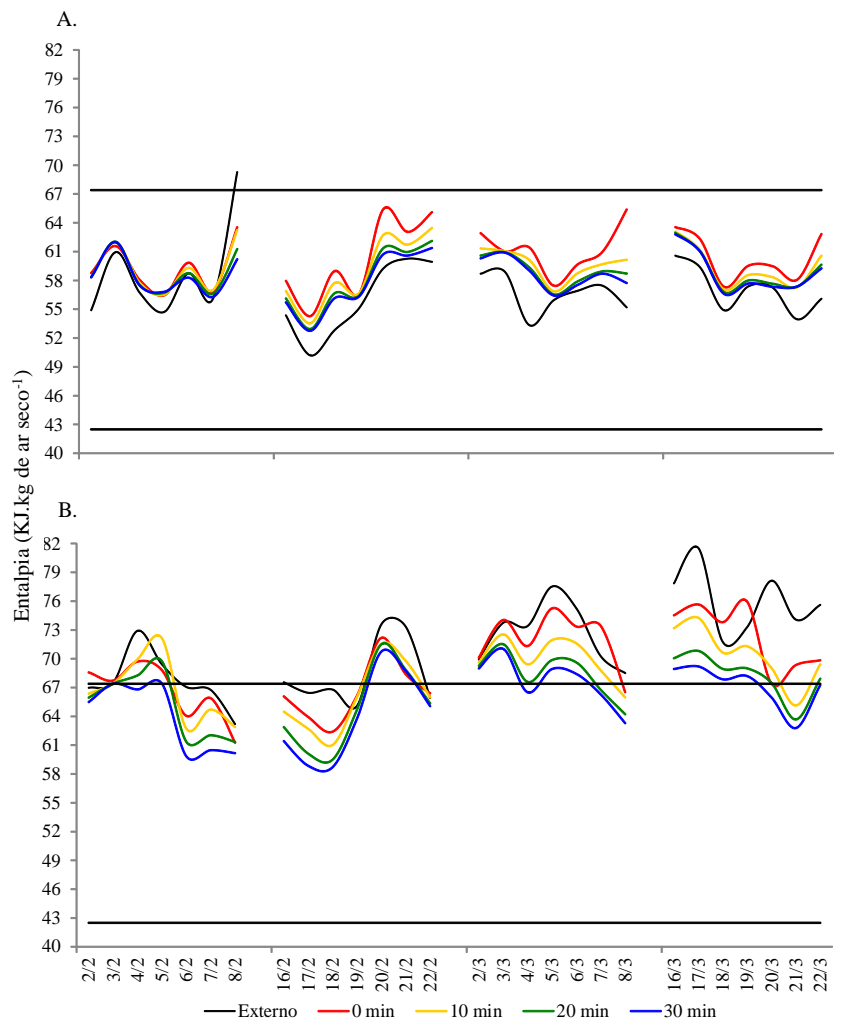

Figura 3. Variação da entalpia nos diferentes tratamentos e no abrigo meteorológico (ambiente externo) no turno da manhã $(A)$ e tarde (B)

No turno da tarde também não se constataram diferenças $(\mathrm{P}>0,05)$ na FR entre os animais dos tratamentos com climatização (10, 20 e 30 min), mostrando efeito significativo $(\mathrm{P}<0,05)$ apenas quando comparados com o sem climatização (0 min), apresentando valores da ordem de 39,5; 37,0; 35,3 e $61,5 \mathrm{mov} \mathrm{min}^{-1}$, respectivamente (Tabela 3 ), resultados que estão de acordo com os estudos realizados por Arcaro Júnior et al. (2005), que também constataram redução na FR de 42,0 para $38,0 \mathrm{mov} \mathrm{min}^{-1}$, para vacas lactantes da raça holandesa, que tiveram acesso ao curral de espera equipado com SRAE, durante 30 min de exposição, na pré-ordenha da tarde.

Segundo Ferreira et al. (2009), para se defende do estresse térmico os bovinos recorrem a mecanismos adaptativos fisiológicos de perda de calor corporal, para evitar a hipertermia;

Tabela 3. Valores médios e desvio padrão dos parâmetros fisiológicos avaliados nos diferentes tratamentos, nos turnos da manhã e da tarde

\begin{tabular}{|c|c|c|c|c|c|}
\hline \multirow{2}{*}{ Variáveis } & \multicolumn{4}{|c|}{ Tratamentos (min) } & \multirow{2}{*}{ CV (\%) } \\
\hline & 0 & 10 & 20 & 30 & \\
\hline \multicolumn{6}{|c|}{ Turno da manhã } \\
\hline $\mathrm{FR}\left(\mathrm{mov} \mathrm{min}^{-1}\right)$ & $36,0 \mathrm{a} \pm 1,51$ & $27,8 b \pm 0,50$ & $28,0 b \pm 0,53$ & $25,8 b \pm 0,50$ & 17,99 \\
\hline $\mathrm{TP}\left({ }^{\circ} \mathrm{C}\right)$ & $30,1 a \pm 1,07$ & $27,7 b \pm 1,05$ & $26,7 \mathrm{c} \pm 1,01$ & $26,5 \mathrm{c} \pm 1,05$ & 06,47 \\
\hline $\operatorname{TR}\left({ }^{\circ} \mathrm{C}\right)$ & $38,2 a \pm 0,16$ & $38,2 \mathrm{a} \pm 0,14$ & $38,1 a \pm 0,18$ & $38,0 a \pm 0,21$ & 00,47 \\
\hline PL (kg) & $10,79 b \pm 0,73$ & $11,00 a b \pm 0,60$ & $11,32 a \pm 0,57$ & $11,43 a \pm 0,70$ & 06,20 \\
\hline \multicolumn{6}{|c|}{ Turno da tarde } \\
\hline $\mathrm{FR}\left(\mathrm{mov} \mathrm{min}^{-1}\right)$ & $61,5 a \pm 13,38$ & $39,5 b \pm 10,24$ & $37,0 b \pm 7,23$ & $35,3 b \pm 8,07$ & 33,31 \\
\hline $\operatorname{TP}\left({ }^{\circ} \mathrm{C}\right)$ & $38,5 a \pm 5,80$ & $31,9 b \pm 2,07$ & $30,8 b \pm 1,60$ & $29,8 b \pm 2,23$ & 14,61 \\
\hline $\operatorname{TR}\left({ }^{\circ} \mathrm{C}\right)$ & $39,1 \mathrm{a} \pm 0,22$ & $38,9 a b \pm 0,21$ & $38,9 a b \pm 0,25$ & $38,7 b \pm 0,16$ & 00,59 \\
\hline PL (kg) & $6,74 a \pm 0,77$ & $6,85 a \pm 0,47$ & $6,87 a \pm 0,51$ & $6,87 a \pm 0,52$ & 08,40 \\
\hline
\end{tabular}

Médias seguidas das mesmas letras nas mesmas linhas não diferem entre si a nível de $5 \%$ de probabilidade, pelo teste de Tukey 
desta forma, aumentam a frequência respiratória, como mecanismo adicional à perda de calor por sudorese constituindo-se, ambos, em meios importantes de perda de calor por evaporação.

Os valores médios da TP apresentaram diferenças significativas $(\mathrm{P}<0,05)$ entre os tratamentos com climatização 20 e $30 \mathrm{~min}\left(26,7\right.$ e $\left.26,5^{\circ} \mathrm{C}\right)$ comparativamente com o tratamento $10 \min \left(27,1^{\circ} \mathrm{C}\right)$ e $0 \min \left(30,1^{\circ} \mathrm{C}\right)$, para o turno da manhã (Tabela 3); no turno da tarde as médias da TP indicaram redução significativa $(\mathrm{P}<0,05)$ nos tratamentos 10,20 e 30 min, quando comparados com o tratamento $0 \mathrm{~min}$, que apresentaram valores da ordem de 31,$9 ; 30,8 ; 29,8$ e $38,5^{\circ} \mathrm{C}$, respectivamente, sem efeito significativo entre os tratamentos com climatização. Os valores médios obtidos nos tratamentos 10,20 e 30 min no turno da tarde, estão ligeiramente abaixo do valor de $32,5^{\circ} \mathrm{C}$ obtido por Perissinotto et al. (2006), para bovinos leiteiros submetidos a climatização.

A temperatura de superfície corporal depende principalmente das condições ambientais de umidade, temperatura do ar e vento e das condições fisiológicas, como vascularização e evaporação pelo suor. Assim, contribui para a manutenção da temperatura corporal mediante trocas de calor com o ambiente em temperaturas amenas e, sob condições de estresse pelo calor, as perdas sensíveis são diminuídas e a evaporação se torna o principal processo de perda de calor (Ferreira et al., 2006).

Em ambos os tratamentos no turno da manhã, a TR se manteve dentro dos valores fisiológicos normais $\left(38\right.$ a $39{ }^{\circ} \mathrm{C}$ ) sugeridos por Du Preez (2000), que não constataram diferenças significativas $(\mathrm{P}>0,05)$ para os valores médios nos animais dos tratamentos $0,10,20$ e $30 \mathrm{~min}$, que indicaram valores da ordem de 38,$2 ; 38,2 ; 38,1$ e $38,0^{\circ} \mathrm{C}$, respectivamente.

Os valores médios no turno da tarde apontaram diferenças significativas $(\mathrm{P}<0,05)$ na TR entre os tratamentos 0 min $(39,1$ $\left.{ }^{\circ} \mathrm{C}\right)$ e $30 \min \left(38,7^{\circ} \mathrm{C}\right)$, com redução de $0,4^{\circ} \mathrm{C}$; verifica-se, ainda, elevação de $0,1{ }^{\circ} \mathrm{C}$ para o tratamento $0 \mathrm{~min}$, sob o limite considerado normal para TR $\left(38\right.$ a $\left.39^{\circ} \mathrm{C}\right)$, sugerido por Du Preez (2000). Resultados semelhantes obtiveram Pinheiro et al. (2005), que trabalharam em sala de espera climatizada com exposição de vacas da raça Jersey em lactação, por $30 \mathrm{~min}$ $\left(38,59{ }^{\circ} \mathrm{C}\right)$ e testemunha sem climatização $\left(39,11{ }^{\circ} \mathrm{C}\right)$ apresentando redução de $\left(0,52^{\circ} \mathrm{C}\right)$.

$\mathrm{O}$ aumento da temperatura retal mostra que os mecanismos de liberação de calor se tornaram insuficientes para manter a homeotermia, porém para os tratamentos 10, 20 e $30 \mathrm{~min}$, no turno da tarde, a TR permaneceu dentro da faixa considerada normal, sinal de que o SRAE foi eficiente na manutenção das respostas fisiológicas dos animais ao acondicionamento do microclima interno da instalação.

Avaliando-se a produção do turno da manhã (Tabela 3) verifica-se diferença significativa $(\mathrm{P}<0,05)$ entre os animais dos tratamentos 20 e $30 \mathrm{~min}$, quando comparada com a do tratamento $0 \mathrm{~min}$. Os animais do tratamento $10 \mathrm{~min}$ não apresentaram diferença significativa quando comparada com a do tratamento 0 min e com as dos tratamentos 20 e $30 \mathrm{~min}$. O aumento na produção, comparativamente com o tratamento sem climatização, foi da ordem de 0,$21 ; 0,53$ e $0,64 \mathrm{~kg}$, correspondente ao acréscimo de 2,2; 4,9 e 5,9\% para os tratamentos 10, 20 e $30 \mathrm{~min}$, respectivamente.
Esses resultados estão de acordo com Barbosa et al. (2004), que encontraram maior produção de leite em vacas que receberam água por aspersão, antes e após a ordenha, $(11,74$ $\left.\mathrm{kg} \mathrm{d}^{-1}\right)$ contra $\left(10,98 \mathrm{~kg} \mathrm{~d}^{-1}\right)$ para as vacas que não receberam aspersão, confirmando o efeito benéfico do umedecimento da superfície corporal no conforto térmico dos animais.

Arcaro Júnior et al. (2003), encontraram acréscimo de 3,45\% na produção diária de leite de vacas holandesas submetidas à climatização no curral de espera, durante 30 minutos, quando comparada a produção com a dos animais que não receberam climatização no curral de espera.

A produção de leite no turno da tarde não apresentou diferença significativa $(\mathrm{P}>0,05)$ entre os tratamentos. $\mathrm{O}$ aumento na produção de leite, comparativamente com o tratamento sem climatização, foi da ordem de 0,$11 ; 0,13$ e 0,13 $\mathrm{kg}$, correspondente ao acréscimo de 1,6;1,8 e 1,8\%, respectivamente, para os animais dos tratamentos 10, 20 e 30 min. Esses resultados divergem dos obtidos por Barbosa et al. (2004) que encontraram diferença significativa na produção de leite de vacas que receberam água por aspersão, antes e após a ordenha $(7,04 \mathrm{~kg})$ contra $(6,74 \mathrm{~kg})$ para as vacas que não receberam aspersão.

Verifica-se, portanto, aumento na produção diária de leite, da ordem de 0,$32 ; 0,66$ e $0,77 \mathrm{~kg} \mathrm{vaca}^{-1} \mathrm{~d}^{-1}$, referente ao acréscimo de 1,$8 ; 3,7$ e $4,4 \%$ para os tratamentos 10,20 e $30 \mathrm{~min}$, respectivamente.

\section{Conclusões}

A exposição dos animais à climatização durante $30 \mathrm{~min}$, possibilitou melhor acondicionamento térmico ambiental, tendo como resposta menores valores para as variáveis fisiológicas: temperatura retal, frequência respiratória e temperatura de pelame, promovendo maior produção de leite por vaca.

\section{LITERATURA CITADA}

Albright, L. D. Environment control for animals and plants. St. Joseph: American Society of Agricultural Engineers Michigan, 1990. 453p. ASAE Textbook, 4

Araújo, A. P. Estudo comparativo de diferentes sistemas de instalações para produção de leite tipo B, com ênfase nos índices de conforto térmico e na caracterização econômica. 2001. 94p. Pirassununga: Universidade de São Paulo. Dissertação Mestrado

Arcaro Júnior, I.; Arcaro, J. R. P.; Pozzi, C. R.; Fagundes, H.; Matarazzo, S. V.; Oliveira, C. A. Teores plasmáticos de hormônios, produção e composição do leite em sala de espera climatizada. Revista Brasileira de Engenharia Agrícola e Ambiental, v.7, p.350-354, 2003.

Arcaro Júnior, I.; Arcaro, J. R. P.; Pozzi, C. R.; Fava, C. D.; Fagundes, H.; Matarazzo, S. V.; Oliveira, J. E. Respostas fisiológicas de vacas em lactação à ventilação e aspersão na sala de espera. Ciência Rural, v.35, p.639-643, 2005. 
Avendano, R. L.; Alvarez, V. F. D.; Correa, C. A.; Saucedo, Q. J. S.; Robinson, P. H.; Fadel, J. G. Effect of cooling Holstein cows during the dry period on posíparíum performance under heat stress conditions. Livestock Production Science, v.105, p.198-206, 2006.

Azevedo, M.; Pires, M. F. A.; Saturnino, H. M.; Lana, A. M. Q.; Sampaio, I. B. M.; Monteiro, J. B. N.; Morato, L. E. Estimativas de níveis críticos superiores do índice de temperatura e umidade para vacas leiteiras 1/2, 3/4 e 7/8 Holandês-Zebú, em lactação. Revista Brasileira de Zootecnia, v.34, p.2000-2008, 2005.

Barbosa, O. R.; Boza, P. R.; Santos, G. T.; Sakagushi, E. S.; Ribas, N. P. Efeitos da sombra e da aspersão de água na produção de leite de vacas da raça Holandesa durante o verão. Acta Scientiarum: Animal Science, v.26, p.115-122, 2004.

Carvalho, V. F.; Yanagi Júnior, T.; Ferreira, L.; Damasceno, F. A.; Silva, M. P. Zoneamento do potencial de uso de sistemas de resfriamento evaporativo no sudeste brasileiro. Revista Brasileira de Engenharia Agrícola e Ambiental, v.13, p.358366, 2009.

Dikmen, S.; Hansen, P. J. Is the temperature-humidity index the best indicator of heat stress in lactating dairy cows in a subtropical environment? Journal of Dairy Science, v.92, p.109-116, 2009.

Du Preez, J. H. Parameters for the determination and evaluation of heat stress in dairy cattle in South Africa. Onderstepoort Journal Veterinary Research, v.67, p.263-271, 2000.

Ferreira, F.; Campos, W. E.; Carvalho, M. F. A; Pires, M. L.; Martinez, M. V. G. B.; Silva, R. S.; Verneques, P. F. Taxa de sudação e parâmetros histológicos de bovinos submetidos ao estresse calórico. Arquivo Brasileiro de Medicina Veterinária e Zootecnia, v.61, p.763-768, 2009.

Ferreira, F.; Pires, M. F. A.; Martinez, M. L.; Coelho, S. G.; Carvalho, A. U.; Ferreira, P. M.; Facury Filho, E. J.; Campos, W. E. Parâmetros fisiológicos de bovinos cruzados submetidos ao estresse calórico. Arquivo Brasileiro de Medicina Veterinária e Zootecnia, v.58, p.732-738, 2006.

Hahn, G. L.; Parkhurrst, A. M.; Gaughan, J. B. Cattle respiration rate as a function of ambient temperature. Transactions of American Society of Agricultural Engineering, v.40, p.97$121,1997$.

Huber, J. T. Alimentação de vacas de alta produção sob condições de stress térmico. In: Bovinocultura leiteira. Piracicaba: FEALQ, 1990. p.33-48.

Martello, L. S.; Savastano Júnior, H.; Pinheiro, M. G.; Silva, S. L.; Roma Júnior, L. C. Avaliação do microclima de instalações para gado de leite com diferentes recursos de climatização. Revista de Engenharia Agrícola, v.24, p.263-273, 2004.
Nääs, I. A.; Arcaro Júnior, I. Influência de ventila e aspersão em sistemas de sombreamento artificial para vacas em lactação em condições de calor. Revista Brasileira de Engenharia Agrícola e Ambiental, v.5, p.139-142, 2001.

Pereira, J. C. C. Fundamentos da bioclimatologia aplicada à produção animal. Belo Horizonte: FEPMVZ, 2005. 195p.

Perissinotto, M.; Moura, D. J. Determinação do conforto térmico de vacas leiteiras utilizando a mineração de dados. Revista Brasileira de Engenharia de Biossistemas, v.1, p.117-126, 2007.

Perissinotto, M.; Moura, D. J.; Cruz, V. F.; Souza, S. R. L.; Lima, K. A. O.; Mendes, A. S. Conforto térmico de bovinos leiteiros confinados em clima subtropical e mediterrâneo pela análise de parâmetros fisiológicos utilizando a teoria dos conjuntos fuzzy. Ciência Rural, v.39, p. 1491-1498, 2009.

Perissinotto, M.; Moura, D. J.; Matarazzo, S. V.; Silva, I. J. O.; Lima, K. A. O. Efeito da utilização de sistemas de climatização nos parâmetros fisiológicos do gado leiteiro. Revista de Engenharia Agrícola, v.26, p.663-671, 2006.

Pinheiro, M. G.; Nogueira, J. R.; Lima, M. L. P.; Leme, P. R.; Macari, M.; Nääs, I. A.; Laloni, L. A.; Titto, E. A. L.; Pereira, A. F. Efeito do ambiente pré-ordenha (sala de espera) sobre a temperatura da pele, a temperatura retal e a produção de leite de bovinos da raça jersey. Revista Portuguesa de Zootecnia, v.12, p.37-43, 2005.

SAS - Statistical analysis system: Realease 6.08 (software), Cary: Sas Institute, 1997. 620p.

Silva, E. V. C. e; Katayama, K. A.; Mace, G. G.; Rueda, P. M.; Abreu, U. G. P.; Ari, C. E. S. N. Z. Efeito do manejo e de variáveis bioclimáticas sobre a taxa de gestação em vacas receptoras de embriões. Ciência Animal, v.11, p.280-291, 2010.

Silva, I. J. O.; Pandorfi, H.; Arcaro Júnior. I.; Piedade, S. M. S.; Moura, D. J. Efeitos da climatização do curral de espera na produção de leite de vacas holandesas. Revista Brasileira de Zootecnia, v.31, p.2036-2042, 2002.

Souza, J. E. R.; Martins Filho, R.; Oliveira, S. M. P.; Neiva, J. N. M.; Lôbo, R. N. B. Influência dos fatores de ambiente no desempenho ponderal de bovinos da raça nelore no Estado do Ceará. Revista Ciência Agronômica, v.34, p.133138, 2003.

Thom, E. C. The discomfort index. Weatherwise, v.12, p.57-59, 1959.

Tinôco, I. F. F. Avicultura industrial: novos conceitos de materiais, concepções e técnicas construtivas disponíveis para galpões avícolas brasileiros. Revista Brasileira de Ciência Avícola, v.3, p.1-26, 2001.

West, J. W. Effects of heat-stress on production in dairy cattle. Journal of Dairy Science, v.86, p.2131-2144, 2003. 